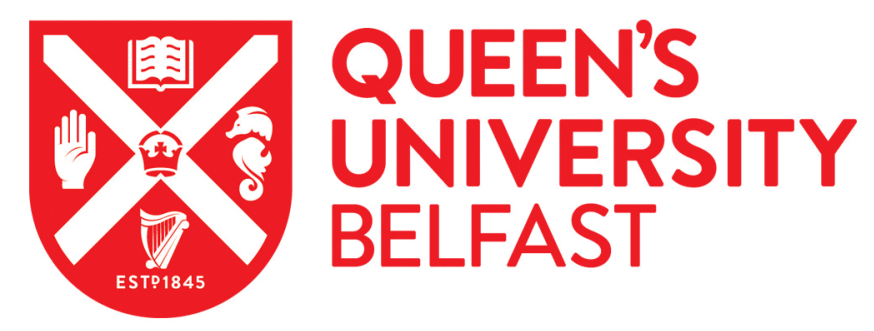

\title{
Positron cooling and annihilation in noble gases
}

Green, D. G. (2017). Positron cooling and annihilation in noble gases. Physical Review Letters, 119(20), [203403]. https://doi.org/10.1103/PhysRevLett.119.203403

\author{
Published in: \\ Physical Review Letters
}

Document Version:

Publisher's PDF, also known as Version of record

Queen's University Belfast - Research Portal:

Link to publication record in Queen's University Belfast Research Portal

Publisher rights

(c) 2017 APS.

This work is made available online in accordance with the publisher's policies. Please refer to any applicable terms of use of the publisher.

\section{General rights}

Copyright for the publications made accessible via the Queen's University Belfast Research Portal is retained by the author(s) and / or other copyright owners and it is a condition of accessing these publications that users recognise and abide by the legal requirements associated with these rights.

Take down policy

The Research Portal is Queen's institutional repository that provides access to Queen's research output. Every effort has been made to ensure that content in the Research Portal does not infringe any person's rights, or applicable UK laws. If you discover content in the Research Portal that you believe breaches copyright or violates any law, please contact openaccess@qub.ac.uk. 


\title{
Positron Cooling and Annihilation in Noble Gases
}

\author{
D. G. Green \\ Centre for Theoretical Atomic, Molecular and Optical Physics, School of Mathematics and Physics, Queen's University Belfast, \\ Belfast BT7 1NN, Northern Ireland, United Kingdom \\ (Received 26 July 2017; published 16 November 2017)
}

\begin{abstract}
Positron cooling and annihilation in room temperature noble gases is simulated using accurate scattering and annihilation cross sections calculated with many-body theory, enabling the first simultaneous probing of the energy dependence of the scattering and annihilation cross sections. A strikingly small fraction of positrons is shown to survive to thermalization: $\sim 0.1$ in $\mathrm{He}, \sim 0$ in $\mathrm{Ne}, \sim 0.15$ in $\mathrm{Ar}, \sim 0.05$ in $\mathrm{Kr}$, and $\sim 0.01$ in Xe. For Xe, the time-varying annihilation rate $\bar{Z}_{\text {eff }}(\tau)$ is shown to be highly sensitive to the depletion of the momentum distribution due to annihilation, conclusively explaining the long-standing discrepancy between gas-cell and trap-based measurements. Overall, the use of the accurate atomic data gives $\bar{Z}_{\text {eff }}(\tau)$ in close agreement with experiment for all noble gases except $\mathrm{Ne}$, the experiment for which is proffered to have suffered from incomplete knowledge of the fraction of positrons surviving to thermalization and/or the presence of impurities.
\end{abstract}

DOI: 10.1103/PhysRevLett.119.203403

The observation of lifetime spectra for positrons annihilating in gases was one of the first sources of information on positron-atom and molecule interactions [1-6]. In particular, measurements of the time-varying normalized annihilation rate $\bar{Z}_{\text {eff }}(t)$ during positron thermalization provided information on the energy dependence of the scattering and annihilation cross sections. Understanding the dynamics of positron cooling, including the fraction of positrons surviving to thermalization, is critical for the accurate interpretation of the positron lifetime experiments. Incomplete thermalization was suspected to be responsible for the lack of consensus among the $\bar{Z}_{\text {eff }}$ data in Xe [7], while modeling of $\bar{Z}_{\text {eff }}(t)[8,9]$ revealed deficiencies in the theoretical data for neon and the heavier noble-gas atoms. Understanding positron kinetics is also crucial for the development of efficient positron cooling in traps and accumulators [10] and for a cryogenically cooled, ultrahigh-energy-resolution, trap-based positron beam $[11,12]$.

Despite the importance of long-standing positroncooling measurements $[5,6]$, there has been a paucity of theoretical studies of positron cooling in gases. Previous studies have mainly employed the diffusion or FokkerPlanck (FP) equation [8,9,13-16]. They used semiempirical or model cross sections, e.g., calculated in the polarizedorbital approximation [17-21], yielding limited success in describing experiments.

Many-body theory (MBT) has provided an accurate and essentially complete description of low-energy positron interactions with noble-gas atoms, taking full account, $a b$ initio, of the strong positron-atom and electron-positron correlations, including virtual-positronium formation [22-25]. Recently, it yielded excellent agreement between theory and experiment for the scattering cross sections, annihilation rates $[24,25]$, and $\gamma$-ray spectra $[25,26]$.
Here, we show that the MBT data enable the accurate modeling of positron cooling and annihilation in noble gases. Using the MBT data in Monte Carlo (MC) simulations [30,31], we calculate the time-evolving positron momentum distribution $f(k, \tau)$ [we work in units where $\tau$ is the time (in nanoseconds) scaled by the number density of the gas $n_{g}$ (in amagat): $\left.\tau=n_{g} t\right]$ and from this the time-varying annihilation rate $\bar{Z}_{\text {eff }}(\tau)=\int Z_{\text {eff }}(k) f(k, \tau) d k / \int f(k, \tau) d k$, which can be determined in experiments. The fraction of positrons surviving to thermalization is shown to be strikingly small, and $\bar{Z}_{\text {eff }}(\tau)$ is shown to be sensitive to the amount of particles annihilating before thermalization, conclusively explaining the long-standing discrepancy between the gas-cell [7] and trap-based [32] $\bar{Z}_{\text {eff }}$ results in Xe. The ab initio calculations enable the first simultaneous probing of the energy dependence of the scattering cross section and annihilation rate. Overall, the MBT-based MC calculations give the best agreement with experiment for all the noble gases to date, except $\mathrm{Ne}$, the experiment for which is proffered to have suffered from the presence of impurities and/or incorrect analysis.

Theory of positron cooling and annihilation.-Below the positronium (Ps)-formation threshold, energy loss in atomic gases proceeds via momentum transfer in elastic collisions. The process of positron thermalization in a Maxwellian gas of temperature $T$ is governed by the mean-squared change in momentum per unit time $\left\langle\Delta k^{2} / \delta \tau\right\rangle=2 B(k)$, where $B(k) \equiv$ $k \sigma_{t}(k) k_{B} T m / M$ [33], $k$ and $m$ are the positron momentum and mass, respectively, $M$ is the mass of the gas atom, and $\sigma_{t}$ is the positron-atom momentum-transfer cross section. It is calculated as $\sigma_{t}=4 \pi k^{-2} \sum_{\ell=0}^{\infty}(\ell+1) \sin ^{2}\left(\delta_{\ell}-\delta_{\ell+1}\right)$ [34], where $\delta_{\ell}(k)$ is the scattering phase shift for a positron of angular momentum $\ell$. Figure 1 (a) shows $B(k)$ for He to Xe, calculated using phase shifts for $\ell=0,1$, and 2 from 

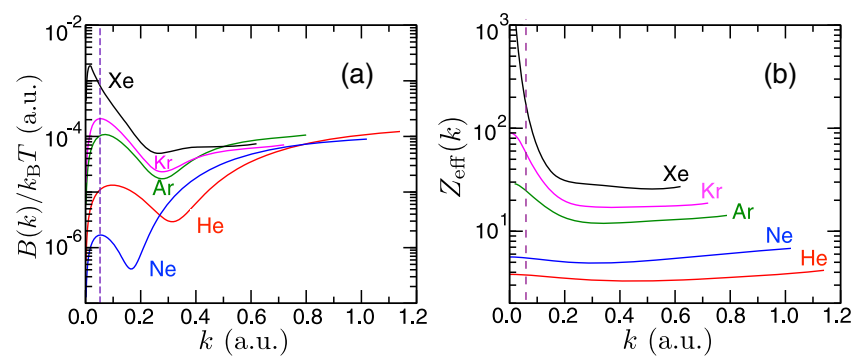

FIG. 1. (a) Momentum-diffusion coefficient $B(k) / k_{B} T=$ $k \sigma_{t}(k) m / M$ vs positron momentum $k$. (b) $Z_{\text {eff }}(k)$ calculated using MBT including $s$-, $p$-, and $d$-wave positrons annihilating on valence and inner valence subshells of the noble gases. Vertical dashed line: thermal positron momentum $k_{\text {th }}=$ $\sqrt{3 k_{B} T} \sim 0.0528$ a.u. at $T=293 \mathrm{~K}$.

MBT [24], with $\ell>2$ partial waves described by the leading $k^{2}$ term in the expansion [35,36]. $B(k)$ exhibits a minimum for all atoms in the sequence He to Xe [Fig. 1(a)], which becomes less pronounced as one moves through the sequence (with the exception of $\mathrm{Ne}$, which has the deepest minimum). As we will see, this leads to "trapping" of positrons and slows down the cooling process in this momentum range.

The annihilation cross section in many-electron targets is parametrized as $\sigma_{a}=\pi r_{0}^{2} Z_{\text {eff }} c / v_{r}[38,39]$, where $r_{0}$ is the classical electron radius, $c$ is the speed of light, $v_{r}$ is the positron speed relative to the target, and $Z_{\text {eff }}$ is the dimensionless effective number of electrons that contribute to the annihilation process. Positron-atom and positronelectron correlations can result in $Z_{\text {eff }}$ being greater than the actual number of valence electrons on which positrons predominantly annihilate [20-24]. $Z_{\text {eff }}(k)$ has been calculated recently for the noble gases via MBT for $s-, p-$, and $d$-wave positrons, taking full account of correlations [24]. The $s, p$, and $d$ waves provide sufficient accuracy to model positron cooling below the Ps-formation threshold: although the contribution of $f$-wave positrons to the total annihilation rate is expected to be $\sim 10 \%$ near the Ps-formation threshold for $\mathrm{Xe}$, we will see that annihilation is unimportant until the positrons have cooled close to the minimum in $B(k)$, at which point the $f$-wave contribution is expected to be negligible $(\sim 1 \%)$ : we note that $Z_{\text {eff }}(k) \sim$ $k^{2 \ell}$ as $k \rightarrow 0$ [24]. From He to Xe, $Z_{\text {eff }}$ becomes increasingly large and strongly peaked at low momenta [Fig. 1(b)]. This effect is due to the existence of positron-atom virtual levels [40], signified by large scattering lengths for Ar, Kr, and Xe (see Table I in Ref. [24]).

Calculation of $f(k, \tau)$. - The momentum $k\left(\tau_{i}\right)$ of an individual positron is determined over an equidistant grid in time-density $\left\{\tau_{i}\right\}$ with step size $\Delta \tau$ as follows. The velocity of a gas atom is sampled from the Maxwell-Boltzmann distribution at $293 \mathrm{~K}$. Both it and the positron velocities are transformed to the center-of-mass frame, in which the energy available for the collision is $E_{\mathrm{CM}}=\mu v_{r}^{2} / 2$, where $\mu$ is the reduced mass. A uniformly distributed random number $r_{1}=U[0,1]$ is drawn, and a collision is deemed to occur if $r_{1}<P=W \Delta \tau$, where $W=v_{r} \sigma_{\mathrm{tot}}\left(E_{\mathrm{CM}}\right)$ is the probability rate of annihilation or elastic collision, with $\sigma_{\text {tot }}=\left(\sigma_{\mathrm{el}}+\sigma_{a}\right)$, subject to the requirement that $P=$ $W \Delta \tau \ll 1$ [41]. Here $\sigma_{\text {el }}$ is the elastic-scattering cross section, which is determined from the integral of the differential cross section $\varrho=d \sigma_{\mathrm{el}} / d \Omega=|f(\theta)|^{2}$, where $f(\theta)$ is the scattering amplitude for scattering angle $\theta$, which is calculated using MBT [see Eq. (31) of Ref. [24]]. If a collision occurs, a second random number $r_{2}=U[0,1]$ is drawn. If $r_{2}<\sigma_{a} / \sigma_{\text {tot }}$, the event is deemed to be annihilation and the particle is removed from the simulation; otherwise, it is an elastic collision and the velocity is updated by sampling $\theta$ by finding the root of $r_{3}=2 \pi \sigma_{\mathrm{el}}^{-1} \int_{0}^{\theta} \varrho \sin \theta^{\prime} d \theta^{\prime}$, where $r_{3}=U[0,1]$ (the azimuthal angle $\phi$ is chosen randomly). The momentum distribution $f(k, \tau)$ results from "binning" positron momenta at each $\tau_{i}$.

Figure 2(a) is an example of $f(k, \tau)$, calculated for $\mathrm{Ar}$ using 50000 positrons initially distributed uniformly in energy up to the Ps-formation threshold. [See Supplemental Material for videos [42] of the evolution of $f(k, \tau)$ for all noble gases.] The initial distribution is seen to quickly evolve $(<100 \mathrm{~ns}$ amg) to a strong Gaussian-like peak near the minimum in $B(k), k_{\min } \sim 0.3$ a.u., which then evolves rather slowly, producing the kneelike feature. This bunching effect becomes less effective as one moves through the sequence from $\mathrm{He}$ to $\mathrm{Xe}$, since the minimum in $B(k)$ becomes less pronounced (with the exception of $\mathrm{Ne}$, which has the deepest minimum). However, even in Xe the formation of a peak in $f(k, \tau)$ at small $\tau$ is evident. Since for any realistic initial distribution most positrons will have initial momenta $k>k_{\min }$, such bunching should be expected, making the overall cooling times somewhat insensitive to the exact form of the initial distribution (see
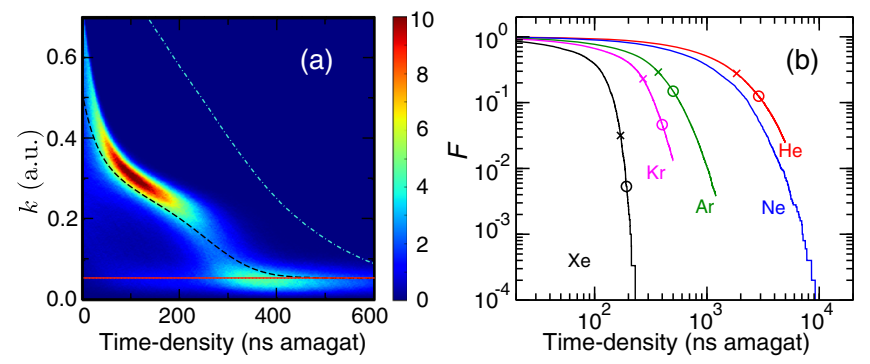

FIG. 2. (a) Density plot of positron momentum distribution $f(k, \tau)$ for Ar, normalized as $\int f(k, \tau) d k=F(\tau)$, the fraction of positrons surviving (dashed-dotted line), calculated using 50000 positrons initially distributed uniformly in energy up to the Ps-formation threshold. Also shown is the rms momentum (black dashed line) and thermal momentum $k_{\mathrm{th}}=\sqrt{3 k_{B} T} \sim 0.0528$ a.u. at $T=293 \mathrm{~K}$ (solid line). (b) Fraction of positrons surviving at time-density $\tau$ (solid lines). Also marked are the values of $F$ at the shoulder time $\tau_{s}$ (crosses) and the complete thermalization time $\tau_{\text {th }}$ (circles). 
below for further details). The small number of positrons with initial momenta below the minimum at early times cool to thermal energies relatively unimpeded, and they form a second, much smaller peak around thermal momentum $k_{\text {th }} \sim \sqrt{3 k_{B} T} \sim 0.0528$ a.u. As the time increases, the Gaussian-like part of the distribution traverses the minimum, roughly maintaining its shape as it does so. As more positrons cool below the minimum, the two peaks in the distribution merge, eventually evolving towards the Maxwell-Boltzmann distribution.

As seen in Fig. 2(a), all but a small fraction of positrons annihilate before the distribution thermalizes at $\tau \sim 400 \mathrm{~ns}$ amg. This fraction is even smaller in other noble gases [see Fig. 2(b)] and has important consequences for the interpretation of measured lifetime spectra (see below). We define the "complete" thermalization time $\tau_{\text {th }}$ as the timedensity at which the rms momentum of the distribution is within $1 \%$ that for a Maxwell-Boltzmann distribution at $293 \mathrm{~K} k_{\text {th }} \sim \sqrt{3 k_{B} T}$. The values of $\tau_{\text {th }}$ are marked in Fig. 2(b) and are presented in Supplemental Table I [42], alongside the fractions of initial positrons remaining at that time-density $F\left(\tau_{\text {th }}\right)$. This fraction is a mere $F\left(\tau_{\text {th }}\right)=0.11$ for $\mathrm{He}$ and reduces by more than an order of magnitude for Xe. Perhaps most remarkably, the fraction of positrons surviving to thermalization in $\mathrm{Ne}$ is practically zero. In $\mathrm{Ne}$, cooling effectively stalls at the minimum of $B(k)$ [see Fig. 1(a)], with positrons eventually succumbing to annihilation (in spite of a relatively small $Z_{\text {eff }} \sim 6$ ) before they can cool further.

Comparison with experiment.-Figures 3(a)-3(e) show $\bar{Z}_{\text {eff }}(\tau)$ for $\mathrm{He}, \mathrm{Ar}, \mathrm{Kr}, \mathrm{Xe}$, and $\mathrm{Ne}$, obtained in calculations that excluded or included particle loss due to annihilation, for positrons initially distributed uniformly in energy, and with initial energy equal to the Ps-formation threshold. The monoenergetic distribution is unphysical but provides an upper limit on the cooling time. For all the noble gases, the increase in $Z_{\text {eff }}(k)$ as $k \rightarrow 0$ results in the evolution of $\bar{Z}_{\text {eff }}(\tau)$ through a transient "shoulder" region resulting from epithermal annihilation at $k<k_{\min }$ [1-3] towards its steady-state thermal value $\bar{Z}_{\text {eff }} \equiv \int_{0}^{\infty} Z_{\text {eff }}(k) f_{T}(k) d k$, where $f_{T}(k)$ is the Maxwell-Boltzmann distribution at $T=293 \mathrm{~K}$. Comparisons between theory and experiment are focused around the shoulder, which is somewhat insensitive to the initial momentum distribution [5,30] [due to the bunching around the minimum in $B(k)$ ]. The traditional measure of the thermalization time in positrongas studies is the "shoulder length" $\tau_{s}$, defined via $\bar{Z}_{\text {eff }}\left(\tau_{s}\right) \equiv \bar{Z}_{\text {eff }}-0.1 \Delta \bar{Z}$, where $\Delta \bar{Z}=\bar{Z}_{\text {eff }}-\bar{Z}_{\text {min }}$ and $\bar{Z}_{\text {min }}$ is the minimum of $\bar{Z}_{\text {eff }}(\tau)$ [43]. The calculated $\tau_{s}$ are given in Supplemental Table I [42], along with
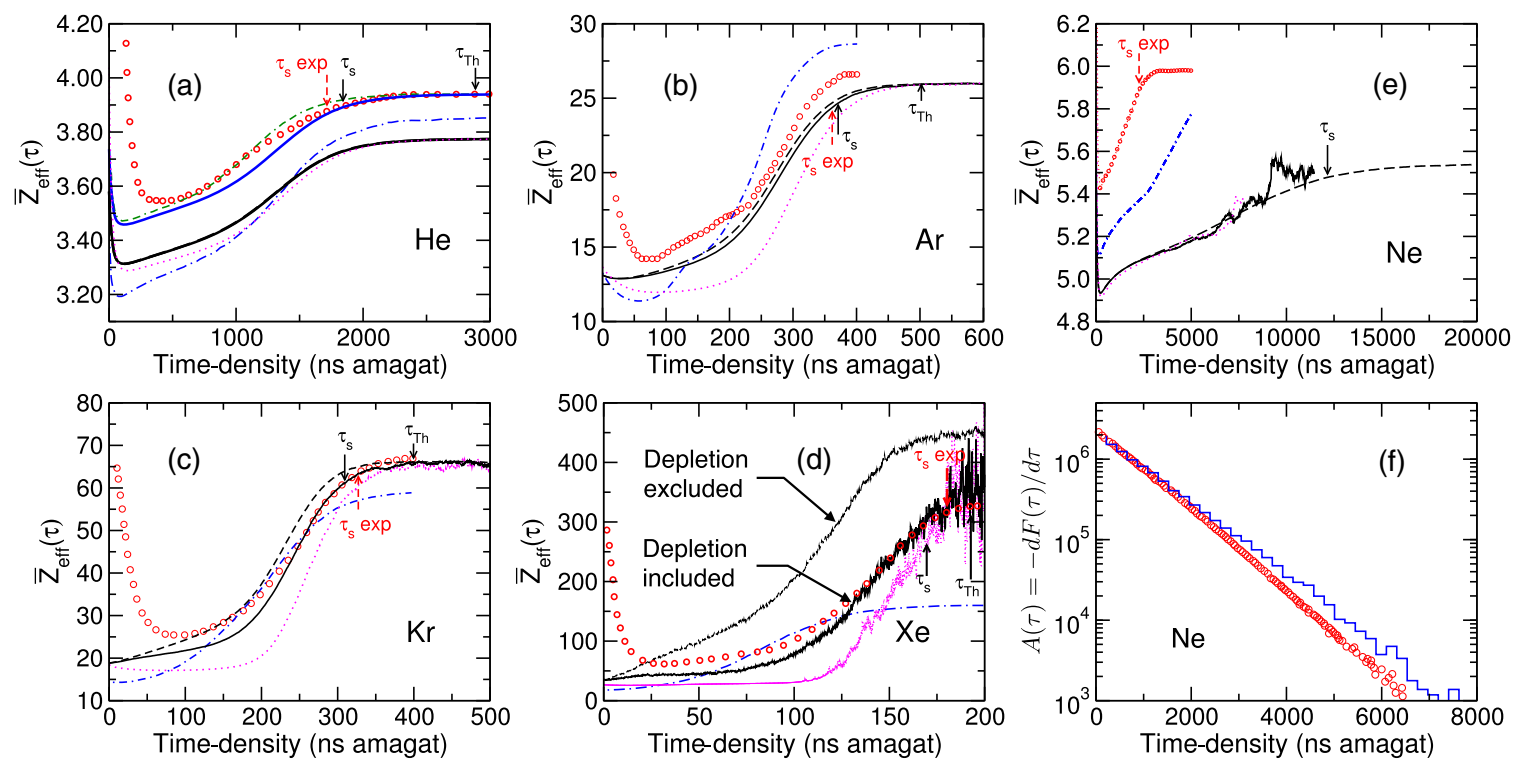

FIG. 3. $\quad \bar{Z}_{\text {eff }}(\tau)$ for He to Xe calculated using $f(k, \tau)$ excluding and including loss of particles due to annihilation, initially distributed uniformly in energy (black dashed and solid lines, respectively), and including annihilation with initial energy equal to the Ps-formation threshold (magenta dotted line). Also shown for He: experiment of Coleman et al. [4] (red circles), present calculation with $\bar{Z}_{\text {eff }}$ scaled to the measured value of $\bar{Z}_{\text {eff }}=3.94$ (blue solid line), FP calculation of Campeanu [14] (blue dashed-dotted line), and model calculations of Boyle et al. [16] scaled to $\bar{Z}_{\text {eff }}=3.94$ (green dash-dash-dotted line); Ar: experiments of Coleman et al. [4,8] (red circles) and FP calculation of Campeanu [8] (blue dashed-dotted line); $\mathrm{Kr}$ and $\mathrm{Xe}$ : experiment of Wright et al. [7] (red circles) and FP calculations of Campeanu [9] (blue dashed-dotted lines); Ne: experiment of Coleman et al. [4] (red circles) and FP calculation of Campeanu [8] (blue dashed-dotted lines). The calculated lifetime spectrum [i.e., observed annihilation rate $A(\tau)=-d F(\tau) / d \tau$ ] for Ne (blue staircase) is also shown (in arbitrary units), compared with experiment [4] (red circles). Black and red arrows mark the calculated and experimental shoulder lengths $\tau_{s}$. 
experimental and previous theoretical results. We now consider the results for each atom in turn, postponing the discussion of $\mathrm{Ne}$ as it is atypical.

Helium.-The calculated $Z_{\text {eff }}(\tau)$ is seen to be insensitive to both the initial distribution and whether depletion of the distribution is included or not. It is known that the MBT slightly underestimates the thermal $\bar{Z}_{\text {eff }}$ in He, predicting a value of $\bar{Z}_{\text {eff }}=3.79$ compared with the experimental value of 3.94 [4]. Scaling the calculated $Z_{\text {eff }}(\tau)$ to the long-time steady-state experimental value, we find excellent agreement with the experiment around the shoulder region, with the calculated shoulder length $\tau_{s}=1839 \mathrm{~ns}$ amg agreeing to within $5 \%$ of the experimental value of $\tau_{s}=1700 \pm 50 \mathrm{~ns}$ amg. The overall shape and length of the calculated shoulder are in better agreement with the experiment than the FP calculation of Campeanu and Humberston [14], who used their Kohn-variational calculated cross sections. The recent diffusion-model calculation of Boyle et al. [16] is also shown. It relied on a carefully tuned model polarization potential and a zerothorder $Z_{\text {eff }}(k)$ scaled by enhancement factors and produced $\tau_{s}=1618 \mathrm{~ns}$ amg. Overall, there is good agreement between the present calculation, that of Boyle et al., and the experiment. This complements the excellent agreement between the MBT and variational calculations and measurements of the elastic-scattering cross sections [24].

Argon.-The present calculated $Z_{\text {eff }}(\tau)$ are sensitive to the initial distribution at small times, but the overall cooling times for both distributions are similar. The result is weakly sensitive to whether depletion of the distribution is included or not. The calculated thermal $\bar{Z}_{\text {eff }}=26.0$ is close to the value of 26.77 measured by Coleman et al. [4]. The calculated shoulder time $\tau_{s}=369 \mathrm{~ns}$ amg is in excellent agreement with the measured value of $362 \pm 5 \mathrm{~ns}$ amg [4], with reasonable overall agreement in the shape of the shoulder. A much smaller shoulder length was measured in the Al Qaradawi experiment, which was suspected to have suffered from the presence of impurities [10]. The present calculations show better agreement with the experiment than the FP calculation of Campeanu [8], which used the polarized-orbital cross section [20].

Krypton.-The calculated shoulder length and $\bar{Z}_{\text {eff }}$ are in excellent agreement with the experiment of Wright et al. [7] (fluctuations at long times are due to the small number of positrons remaining). The FP calculation of Campeanu [9], which used the polarized-orbital cross sections [21], underestimates $\bar{Z}_{\text {eff }}$.

Xenon.-The case of $\mathrm{Xe}$ is special because of the strong peaking of $Z_{\text {eff }}$ at small $k$, which means that annihilation successfully competes with cooling at epithermal positron momenta. When positron depletion due to annihilation is neglected, the positron cooling is fast $\left(\tau_{s} \sim 150 \mathrm{~ns} \mathrm{amg}\right)$, and $\bar{Z}_{\text {eff }}$ plateaus at $\sim 450$. Including depletion brings the shoulder region and shoulder length into excellent agreement with the experiment [7] (fluctuations are due to the small fraction of positrons remaining). The FP calculation of Campeanu is in serious disagreement with the experiment and the MBT result. It used the model polarizedorbital cross sections of McEachran [21], which predict elastic-scattering cross sections and $Z_{\text {eff }}(k)$ that are smaller than the MBT calculation and experiment [24].

The present calculations show that $\bar{Z}_{\text {eff }}$ is highly sensitive to the loss of particles due to annihilation. The vigorous increase in $Z_{\text {eff }}(k)$ as $k \rightarrow 0$ leads to a quasi-steady-state long-time distribution whose low-momentum component is found to be suppressed relative to the Maxwell-Boltzmann one and a steady-state annihilation rate $Z_{\text {eff }}(\tau \rightarrow \infty) \sim 350$ that is significantly reduced from the calculated true thermal $\bar{Z}_{\text {eff }} \sim 450$. The present results thus conclusively explain the discrepancy between the gas-cell measurement of $\bar{Z}_{\text {eff }} \sim 320$ of Wright et al. [7] and the PenningMalmberg trap measurement $\bar{Z}_{\text {eff }} \sim 401$ of the Surko group [32], whose setup ensures positrons are well thermalized. We remark that by adding small amounts of a lighter, low$Z_{\text {eff }}$ gas, e.g., $\mathrm{He}$ or $\mathrm{H}_{2}$, to Xe, Wright et al. measured an increase of the pure Xe $\bar{Z}_{\text {eff }} \sim 320$ to $\bar{Z}_{\text {eff }}=400-450$ [7], which is broadly consistent with the present calculated value and the Surko-group measurement. The mechanism for such an increase with an admixture of $\mathrm{He}$ is, however, unclear, given that momentum transfer is more effective in $\mathrm{Xe}$ as $k \rightarrow 0$ [see Fig. 1(a)].

Neon.-The calculated shoulder time (in this case calculated excluding the loss of particles due to annihilation) is $\tau_{s}=12000 \mathrm{~ns}$ amg, by which time the fraction of positrons remaining is practically zero [see Fig. 3(e)]. It is drastically longer than the measured value $\tau=2700 \mathrm{~ns}$ amg [4] (note that the FP calculation of Campeanu [8] is also slower and has not reached a steady-state value). This serious disagreement is in spite of the good agreement of the MBT elasticscattering cross section with the experiment, including at the Ramsauer minimum [24]. Moreover, the present calculation is consistent with that expected from mass scaling the He result (which has a similar sized $\sigma_{t}$ ) $\tau_{\mathrm{th}}^{\mathrm{He}} M_{\mathrm{Ne}} /$ $M_{\mathrm{He}} \sim 5 \tau_{\mathrm{th}}^{\mathrm{He}} \sim 14500 \mathrm{~ns}$ amg.

The experimental shoulder length was determined by Coleman et al. [4] via straight line fits to the lifetime spectrum. However, in spite of the serious discrepancy in $\tau_{s}$, the calculated and measured lifetime spectra are in surprisingly good agreement [see Fig. 3(f)]. Importantly, the calculated $\tau_{s}=12000 \mathrm{~ns}$ amg and $\tau_{\text {th }} \sim 21000 \mathrm{~ns}$ amg are much longer than the $0-8000 \mathrm{~ns}$ amg considered in the experimental analysis. As seen in Fig. 2(b), at $\tau \lesssim$ $8000 \mathrm{~ns}$ amg the vast majority of positrons have already annihilated, after cooling had effectively stalled around the minimum in $B(k)$. Since $Z_{\text {eff }}(k)$ is a reasonably flat function around the minimum, a signal of many to all of the positrons annihilating at momenta close to the minimum would be observed as a leveling off of $\bar{Z}_{\text {eff }}(\tau)$, which could have erroneously been interpreted as the true thermal $\bar{Z}_{\text {eff }}$. A second possible source of error in the experimental 
determination of the shoulder length is that it was affected by the presence of impurities. It is known that positron cooling in gases like $\mathrm{CO}_{2}$ and $\mathrm{N}_{2} \mathrm{O}$ is fast, e.g., $0.1 \mathrm{~ns}$ amg [10]. The presence of even minute amounts of impurities could thus lead to a significant reduction in the cooling time.

Summary.-Positron cooling in noble gases has been simulated using accurate cross sections calculated $a b$ initio from many-body theory. The fraction of positrons surviving to thermalization was shown to be strikingly small. For Xe, the time-varying dimensionless annihilation rate $\bar{Z}_{\text {eff }}(\tau)$ is shown to be strongly affected by the depletion of positrons, conclusively explaining the long-standing discrepancy between gas-cell [7] and trap-based [32] measurements in Xe. Overall, the use of the accurate atomic data gives the best agreement to date with experiment for all noble gases except $\mathrm{Ne}$, the experiment for which is proffered to have suffered from incomplete knowledge of the fraction of positrons surviving to thermalization and/or the presence of impurities. New lifetime-spectra measurements, or alternatively measurements of the time-varying annihilation $\gamma$ spectra [44], are now warranted.

Data relating to this article can be accessed online [45].

I thank Gleb Gribakin for valuable discussions and for carefully reading the manuscript and Patrick Mullan, Mike Charlton, and Chris Harvey for valuable discussions. This work was supported by the United Kingdom EPSRC, Grant No. EP/N007948/1.

*d.green@qub.ac.uk

[1] W. R. Falk and G. Jones, Can. J. Phys. 42, 1751 (1964).

[2] S. J. Tao, J. Bell, and J. H. Green, Proc. Phys. Soc. London 83, 453 (1964).

[3] D. A. L. Paul, Proc. Phys. Soc. London 84, 563 (1964).

[4] P. G. Coleman, T. C. Griffith, G. R. Heyland, and T. L. Killeen, J. Phys. B 8, 1734 (1975).

[5] T. C. Griffith and G. R. Heyland, Phys. Rep. 39, 169 (1978).

[6] M. Charlton, Rep. Prog. Phys. 48, 737 (1985).

[7] G. L. Wright, M. Charlton, T. C. Griffith, and G. R. Heyland, J. Phys. B 18, 4327 (1985).

[8] R. I. Campeanu, J. Phys. B 14, L157 (1981).

[9] R. I. Campeanu, Can. J. Phys. 60, 615 (1982).

[10] I. Al-Qaradawi, M. Charlton, I. Borozan, R. Whitehead, and I. Borozan, J. Phys. B 33, 2725 (2000).

[11] M. R. Natisin, J. R. Danielson, and C. M. Surko, J. Phys. B 47, 225209 (2014).

[12] M. R. Natisin, J. R. Danielson, and C. M. Surko, Appl. Phys. Lett. 108, 024102 (2016).

[13] P. H. R. Orth and G. Jones, Phys. Rev. 183, 16 (1969).

[14] R. I. Campeanu and J. W. Humberston, J. Phys. B 10, 239 (1977).

[15] B. Shizgal and K. Ness, J. Phys. B 20, 847 (1987).

[16] G. J. Boyle, M. J. E. Casey, R. D. White, and J. Mitroy, Phys. Rev. A 89, 022712 (2014).

[17] R. P. McEachran, A. G. Ryman, A. D. Stauffer, and D. L. Morgan, J. Phys. B 10, 663 (1977).
[18] R. P. McEachran, D. L. Morgan, A. G. Ryman, and A. D. Stauffer, J. Phys. B 11, 951 (1978).

[19] R. P. McEachran, A. G. Ryman, and A. D. Stauffer, J. Phys. B 11, 551 (1978).

[20] R. P. McEachran, A. G. Ryman, and A. D. Stauffer, J. Phys. B 12, 1031 (1979).

[21] R. P. McEachran, A. D. Stauffer, and L. E. M. Campbell, J. Phys. B 13, 1281 (1980).

[22] V. A. Dzuba, V. V. Flambaum, W. A. King, B. N. Miller, and O. P. Sushkov, Phys. Scripta T46, 248 (1993).

[23] V. A. Dzuba, V. V. Flambaum, G. F. Gribakin, and W. A. King, J. Phys. B 29, 3151 (1996).

[24] D. G. Green, J. A. Ludlow, and G. F. Gribakin, Phys. Rev. A 90, 032712 (2014).

[25] D. G. Green and G. F. Gribakin, Phys. Rev. Lett. 114, 093201 (2015).

[26] MBT was also applied to positron interactions with hydrogenlike ions [27] and to study the effect of positron-atom correlations on positron-molecule $\gamma$-ray spectra [28,29].

[27] D. G. Green and G. F. Gribakin, Phys. Rev. A 88, 032708 (2013).

[28] D. G. Green, S. Saha, F. Wang, G. F. Gribakin, and C. M. Surko, New J. Phys. 14, 035021 (2012).

[29] D. G. Green, S. Saha, F. Wang, G. F. Gribakin, and C. M. Surko, Mater. Sci. Forum 666, 21 (2010).

[30] A. Farazdel and I. R. Epstein, Phys. Rev. A 16, 518 (1977).

[31] D. G. Green (to be published).

[32] T. J. Murphy and C. M. Surko, J. Phys. B 23, L727 (1990).

[33] E. M. Lifshitz and L.P. Pitaevskii, Physical Kinetics, Course of Theoretical Physics Vol. 10 (Pergamon, Oxford, 1981).

[34] L. D. Landau and E. M. Lifshitz, Quantum Mechanics (Nonrelativistic Theory), 3rd ed., Course of Theoretical Physics Vol. 3 (Pergamon, Oxford, 1977).

[35] T. F. O’Malley, L. Spruch, and L. Rosenberg, J. Math. Phys. (N.Y.) 2, 491 (1961).

[36] Higher-order terms in $\delta_{\ell}$ for $\ell>2$ [37] contribute negligibly to $\sigma_{t}$, e.g., $<3 \%$ in Xe at the Ps-formation threshold, quickly reducing to $<1 \%$ at $k<0.55$ a.u., and can thus be neglected.

[37] M. K. Ali and P. A. Fraser, J. Phys. B 10, 3091 (1977).

[38] P. A. Fraser, Adv. At. Mol. Phys. 4, 63 (1968).

[39] I. Pomeranchuk, Zh. Eksp. Teor. Fiz. 19, 183 (1949).

[40] V. I. Goldanski and Y. S. Sayasov, Phys. Lett. 13, 300 (1968).

[41] In practice, we demand that $P=W \Delta t<0.1$.

[42] See Supplemental Material at http://link.aps.org/ supplemental/10.1103/PhysRevLett.119.203403 for (i) a table comparing the present calculated thermalization times and shoulder lengths with previous calculated and measured values for $\mathrm{He}, \mathrm{Ne}, \mathrm{Ar}, \mathrm{Kr}$, and $\mathrm{Xe}$, and (ii) links to videos showing evolution of various quantities, e.g., the positron momentum distribution, during the cooling process for $\mathrm{He}$, $\mathrm{Ne}, \mathrm{Ar}, \mathrm{Kr}$ and $\mathrm{Xe}$.

[43] D. A. L. Paul and C. Y. Leung, Can. J. Phys. 46, 2779 (1968).

[44] D. G. Green, following Letter, Phys. Rev. Lett. 119, 203404 (2017).

[45] http://doi.org/10.17034/9da6ab49-3a41-4042-90edf7e $479 \mathrm{~d} 2 \mathrm{f} 437$. 